\title{
Klinischer Kompetenzimport als strategisches Management gegen MDR-Disruption
}

\author{
HPZenner Clinical ${ }^{1}$ \\ ${ }^{1}$ Affiliation not available
}

June 1, 2020

\section{Jannik Pieper und Hans-Peter Zenner}

Jannik Pieper, M.Sc., Associate, Prof. Dr. med. Hans-Peter Zenner, MBA , HPZenner Clinical Research Organization, Tübingen

\section{Zusammenfassung}

Durch die Einführung der neuen EU-Medizinprodukte-Verordnung sehen sich Unternehmen einer disruptiven Kraft gegenüber. Der deutlich umfassendere Nachweis klinischer Daten ausnahmslos mittels Klinischer Bewertung (CER, Clinical evaluation Report) gem. MEDDEV 2.7/1 rev. 4 ist für Unternehmen ein neuartiger Prozess und verändert die Wertschöpfung der Unternehmen. Liegt kein ordnungsgemässer CER vor, sind keine EU-Konformitätserklärung bzw. CE-Zertifizierung und damit keine Vermarktung möglich. Fehlende Compliance von Unternehmen entfaltet damit disruptive Kraft, denn sie führt zu Marktausschlüssen von Medizinprodukten bis hin zur Schließung ganzer Unternehmen.

\section{Einführung}

Die neue MDR (EU-Medizinprodukte-Verordnung, 2017/74511MDD 2017/745) führt auf regulatorischem Wege zu einer Disruption des Marktes für Medizinprodukte. Sie greift ab dem 26. Mai 2021. Seit 31.12.18 ist bereits die Leitlinie MEDDEV 2.7/1 revision 4 verbindlich, die die Abfassung von Klinischen Bewertungen gem. MDR beschreibt. Fehlende Compliance von Unternehmen entfaltet disruptive Kraft, denn sie führt zu Marktausschlüssen von Medizinprodukten bis hin zur Schließung ganzer Unternehmen (Eckert, 2014). Hauptgrund einer MDR-Non-Compliance mit Ausschluss vom Markt kann eine fehlende oder nicht ordnungsgemäße neuartige Klinische Bewertung (CER, Clinical Evaluation Report) oder Klinische Prüfung gemäß MDR sein. Nicht ordnungsgemäß kann ein CER wegen formaler als auch inhaltlicher Mängel oder/und auf Grund fehlender Voraussetzungen des Autors sein.

Spätestens mit dem 26. Mai 2021 müssen Hersteller zum erstmaligen Inverkehrbringen von Medizinprodukten das EU-Zertifikat (bzw. die Konformitätserklärung) nach der neuen MDR vorlegen (Artikel 35, EU-Medizinprodukte-Verordnung, 2017, geänderte Fassung von 2020). Gleiches gilt für die Erneuerung von CE-Zertifikat bzw. Konformitätserklärung. Das Verfahren umfasst ausnahmslos eine Klinische Bewertung und diese dazu noch in einer neuartigen Qualität und im Einzelfall sogar mit einer Klinische Prüfung. Der Klinische Bewerter sollte die deutlich erhöhten Anforderungen gem. Artikel 6.4 MEDDEV 2.7/1 rev 4 erfüllen. Liegt kein ordnungsgemässer CER vor, sind keine EU-Konformitätserklärung bzw. CE-Zertifizierung und damit keine Vermarktung möglich.

Mit Blick auf Klinische Bewertung und Prüfung finden sich die Neuerungen der MDR (EU-Verordnung 2017/745) im Vergleich zur alten MDD (EU-Richtlinie 93/42/EWG) v.a. in folgenden Bereichen:

1. Eine klinische Bewertung wird ausnahmslos erforderlich. 
2. Massiv erhöhte Qualitätsanforderungen an die klinische Bewertung. Diese gehen zurück auf erhöhte Anforderungen an klinische Sicherheit und klinischen Leistungs-Nachweis.

3. Deutlich erhöhte Qualifikationsanforderungen an die Klinischen Bewerter. In der Regel wird Facharztstandard gefordert.

4. Zunahme der Zahl Klinischer Prüfungen gemäß EN ISO 14155. Neuartige Produkte, implantierbare Medizinprodukte und Produkte der Klasse III müssen grundsätzlich eine klinische Prüfung durchlaufen.

5. Klinische Überwachung nach dem Inverkehrbringen (klinische PMS)

\section{Material und Methoden}

Es wurde eine systematische Recherche in den Datenbanken EUR Lex und Pubmed durchgeführt. Schlüsselwörter wurden in die Suchprotokolle aufgenommen. Basierend auf der Cochrane Highly Sensitive Search Strategy (HSSS) wurde eine hochmoderne Suchstrategie angewendet, die eine hohe Sensitivität beibehält, aber die Präzision beim Abrufen von Berichten verbessert, z.B. in der PubMed-Version von Medline. Einzelne Begriffe und Operatoren wurden Term für Term hinzugefügt, um Berichte zu identifizieren. Nachfolgend ein Beispiel für eine Suchstrategie in Pubmed mit Text (alle Felder; Überschriften; Unterüberschriften) und MeSH-Schlüsselwörtern sowie Booleschen Operatoren.

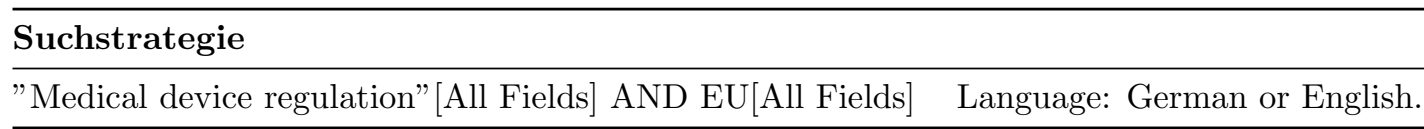

Tab.1 Beispiel einer Datenbank-Suchstrategie mit Freitext-Schlüsselwörtern und/oder Schlagworten (MeSH), Booleschen Operatoren

\section{Ergebnisse und Diskussion}

Unternehmensrisiken durch disruptive Kräfte der MDR

Die eingangs genannten klinischen Anforderungen der MDR sind für Medizinproduktehersteller hohe und unvermeidbare Unternehmensrisiken. Denn anders als forschende Pharmaunternehmen beschäftigen Medizinproduktehersteller häufig kein eigenes Fachpersonal (z.B. Ärzte), dass den von der MEDDEV 2.7/1 rev. dargelegten Facharztstandard erfüllt.

Disruption bezeichnet einen Umbruch/ eine Veränderung. Aufmerksamkeit für diesen Begriff wurde erstmals durch Clayten 1997 mit der Vorstellung seiner ,,theory of disruptive innovation“ erzeugt. Die Einfuhrung der MDR bedeutet zweifelsohne ebenfalls eine Disruption fur Unternehmen in den betroffenen Markten. Durch einen Markteingriff in Form eines EU-Gesetzes werden die am Markt teilnehmenden Unternehmen zu neuartigem Handeln gezwungen.

Im Gegensatz zu anderen externen Disruptionen, wie etwa die Finanzkrise 2007 oder die Corona-Krise 2020, konnten und konnen sich Unternehmen auf das Inkrafttreten des Gesetzes vorbereiten. Die Folgen fur die Unternehmen unterscheiden sich dabei von der Art und der Tiefe des Eingriffs in ihr Unternehmen. Die Disruption, die die Einfuhrung des MDR mit sich bringt, verandert die Prozesse der Produktion eines Produkts und der Produktinnovation wahrend zugleich durch den Mehraufwand hohere Kosten hinzukommen. Durch den Eingriff der Europaischen Union werden disruptive Krafte ausgelost, die die Sicherheit von medizinischen Produkten verbessern sollen.

SWOT-analytisch kann man dieses MDR-Markrisiko den Starken und Schwachen des Unternehmens gegenuberstellen. Dabei fallt ins Auge, dass Unternehmen mit der Schwache, die klinischen Anforderungen der MDR an Qualitat und Qualifikation nicht zu erfullen (Tabelle 1), die Unterbrechung ihrer Wertschopfungskette bis hin zum Ausscheiden aus dem Markt droht. Der jedes Unternehmen treffende Zwang der MDR zum klinischen Nachweis der Evidenz der Wirksamkeit von Medizinprodukten ist damit ein disruptiver regulatorischer Eingriff in den Markt, der zur Marktbereinigung fuhrt (Gans, 2016).

Unternehmensstarke und verbesserte Marktpenetranz durch Implementierung klinischer Prozesse 
Hersteller mit der Managementstarke, MDR-Resilienz u.a. durch klinische Expertise in ihr Unternehmen zu implementieren, werden hingegen nicht nur weiter am Markt bleiben, sondern sie werden auf eine Marktbereinigung mit sinkender Zahl von Mitbewerbern treffen (Bain 1962). Der Import klinischer Expertise kann damit sowohl Treiber MDR-complianter Unternehmensstarke als auch von hoheren Marktanteilen und Wachstum sein.

Unternehmen, die dem disruptiven MDR-Risiko mit der Entwicklung von klinischer MDR-Resilienz als geeigneter Unternehmensstarke begegnen wollen, mussen daher auf die klinischen Anforderungen der MDR reagieren. Sie transformieren ihre Wertschopfungskette durch die ihnen bisher in der Regel nicht bekannte Einbeziehung klinischer Expertise gemass Facharztstandard.

Eine Moglichkeit ist die Beschaftigung von evaluationserfahrenen Fachkraften, wie z.B. von Facharzten, die die Qualifikationsanforderungen gemass Art. 6.4 MEDDEV 2.7/1 Revision 4 erfullen. Freilich kann die Implementierung klinischer Prozesse als in-house Expertise zu lange dauern, zu aufwandig sein und am Schluss sogar misslingen (Bruch 2013). Ursache ist beispielsweise der Mangel an geeigneten Facharzten auf dem Personalmarkt. Hinzu kommt, dass der zusatzliche Aufwand als Fixkosten, voraussichtlich sogar in Form von Sprungfixkosten, anfallt, die bei einem Misslingen nicht leicht abgeschmolzen werden konnen.

\begin{tabular}{ll}
\hline Klinische Verfahren gem. MDR & Mögliche klinische Defizite \\
\hline Klinische Bewertung & Keine klinische Expertise \\
Literaturverfahren & Keine Befähigung zur Volltextbewertung \\
Äquvalenzverfahren & Kein Zugang zu Unterlagen des Mitbewerbers \\
Klinischer Evaluationsplan & Neu; keine klinische Expertise \\
Klinischer Entwicklungsplan & Neu; keine klinische Expertise \\
Klinische Studien & Keine Erfahrung; keine klinische Expertise \\
Klinische Evaluatoren & Keine fachärztlichen Evaluatoren \\
Post Market Surveillance & Verschärft, keine klinische Expertise \\
\hline
\end{tabular}

Tabelle 2: MDR-bedingte Wertschöpfungsblockaden im Unternehmen mit Schwächen durch fehlende klinische Expertise

Eine Alternative ist klinisches Outsourcing, und zwar an CROs (Clinical/Contract Research Organization), deren Klinische Evaluatoren z.B. Fachärzte sind. Die Fachärzte der CRO müssen naturgemäß die Klinische Bewertung gemäß MDR/ MEDDEV 2.7/1 Revision 4 und die klinische Studienplanung gemäß EN ISO 14155 beherrschen (EU-Medizinprodukte-Verordnung, Artikel 15, 2017). Als evaluationserfahrene Fachärzte ergänzen sie das technische Wissen von Medizinprodukteherstellern um die zwingend notwendige klinische Expertise und stehen in der Regel zeitnah zur Verfügung. Eine derartig aufgestellte CRO unterstützt Medizinprodukteunternehmen bei der Transformation ihrer Wertschöpfungsketten an die MDR. Neben dem Import der notwendigen klinischen Expertise handelt es sich darüber hinaus um eine agile Management-Methode zur Delegation von Workload, zur Einhaltung der Zeitachse und zur Kostenplanung (Maximini, 2018). Es entstehen nämlich nur variable Kosten, keine (Sprung)-Fixkosten.

Eine CRO kann beauftragt werden, den gesamten Klinischen Bewertungsbericht gemäß MDR und nach Facharztstandard zu erstellen. Nachfolgend werden wesentliche nach MDR vorgeschriebene Themen, die Teil einer MDR-konformen Klinischen Bewertung sind, aufgeführt:

- Klinischer Bewertungsplan

- Klinischer Entwicklungsplan

- Analyse präklinischer Daten

- Analyse klinischer Daten

- Literaturverfahren

- Äquivalenzverfahren

- Klinische Konformitätsprüfung 
- Gap-Analyse

- Ethische Risiko-Nutzen-Bewertung

- Klinischer Bewertungsreport (CER)

- $\mathrm{PMS} / \mathrm{PMCF}$

Je nach CRO können häufig auch einzelne Arbeitspakete gebucht werden. Ein vollständiger klinischer Bewertungsreport ist ein aufwändiges und sehr umfangreiches Dokument, wenn dieser Report gemäß der neuen MDR nach MEDDEV 2.7/1 Revision 4 ausgeführt wird. Um Outsourcing-Kosten zu minimieren, bieten einzelne CROs deshalb an, nur einzelne Arbeitspakete erstellen zu lassen.

Einzelne Arbeitspakete können noch weiter verbilligt werden, wenn mehrere Hersteller gleichartiger Medizinprodukte wesentliche Teile des Literatur- und State-of-the-Art-Verfahrens gemeinsam beauftragen. Dieses Sharing-Verfahren stellt eine weitere Vorgehensweise dar, die Kosten bei der Erstellung Klinischer Bewertungen gemäß MDR weiter zu reduzieren.

\section{Klinische Prüfungen von Medizinprodukten}

Liegen keine ausreichenden klinischen Nachweise vor, um die geforderte klinische Sicherheit und Leistung eines Produkts zu beweisen, müssen klinische Prüfungen durchgeführt werden. Neuartige Produkte, implantierbare Medizinprodukte und Produkte der Klasse III müssen grundsätzlich eine klinische Prüfung durchlaufen. Eine klinische Prüfung ist ausnahmslos durchzuführen bei

- Neuer Indikation

- Neuer anatomischer Region des Körpers

- Änderungen eines in den Verkehr gebrachten Produkts mit möglicherweise maßgeblichem Einfluss auf Sicherheit oder Wirksamkeit

- Maßgeblicher Verlängerung der Anwendungszeit

- Unzureichender Literaturlage zu Wirksamkeit und Risiken

Klinische Studien mit Medizinprodukten müssen gemäß EN ISO 14155 ,,Klinische Prufung von Medizinprodukten an Menschen - gute klinische Praxis" sowie weiterer Vorschriften geplant und durchgefuhrt werden. Hier gelten weitere Vorschriften wie die VO uber klinische Prufung vom Medizinprodukten und die Medizinprodukte-Sicherheitsplanverordnung (MPSV). Hingegen entfallen nach MDR die SSSS20ff. des Medizinproduktegesetzes sowie die Medizinprodukte klinische Prufverordnung MPKPV. Die Anforderungen der EN ISO 14155 sind mit der ICH-GCP fur klinische Studien mit Arzneimitteln vergleichbar.

Die Durchfuhrung klinischer Prufungen von Medizinprodukten und IVD benotigt nach SS 20 Abs. 1 MPG eine Genehmigung durch die zustandige BoB BfArM/PEI und die zustimmende Bewertung durch die zustandige offentlich-rechtliche EK z.B. bei Arztekammern oder Universitatsklinika. Antrage sind uber das DIMDI/BfArM-Portal zu stellen.

Bei der Erfullung der Anforderungen an die arztliche Durchfuhrung klinischer Prufungen von Medizinprodukten konnen CROs den Unternehmer als Sponsor unterstutzen, und zwar durch Erstellung der Investigator's Brochure, des Studienprotokolls als auch durch die Erstellung von Aufklarungs- und Einwilligungserkarungstexten fur Patienten. Hinzu konnen die Aufstellung des Prufplans nach DIN EN ISO 14155 sowie die klinische Bewertung der Ergebnisse kommen.

\section{Literatur}

1. Bain, J.S.: Barriers to New Competition. Buch 1962.

2. Bruch, H.: Outsourcing: Konzepte und Strategien, Chancen und Risiken. Springer 2013

3. Christensen, C. M.: The Innovator's Dilemma . Harvard 1997

4. Dimachkie Masri, M., Ramirez, B., Popescu, C. and Reggie, E. (2012), "Contract research organizations: an industry analysis", International Journal of Pharmaceutical and Healthcare Marketing, Vol. 6 No. 4, pp. 336-350. https://doi.org/10.1108/17506121211283226

5. Eckert, T., 2014. Praxiswissen Compliance: erfolgreiche Umsetzung im Unternehmen. HaufeLexware. (Seite 20-21)

6. Gans, J., 2016. The Disruption Dilemma, The MIT Press. MIT Press. (Seite 9-10)

7. Maximini, D., 2018. Agile Leadership in Practice: Applying Management 3.0. 


\section{IMPRESSUM}

HPZenner Publishing Tübingen

Geschäftsbereich der H.P. Zenner Clinical GmbH\&CoKG

HRB 765428 AG Stuttgart

Bismarckstr. 66, 72072 Tübingen

+497071 49-645 office@hpzenner.de

Geschäftsführer: Prof. Dr. H.P. Zenner

(C) H.P. Zenner Clinical GmbH\&CoKG

This report is subject to the protection by the German Copyright Law $\S \S 1,2,11,15$ UrhG from 09.09.1965, BGBI, I S, 1273 (revision from 09.06.1993 BGBI, S. 910) of the copyright law. 